\title{
Comparing VR Environments for Seat Selection in an Opera Theater
}

\author{
José Luis Dorado ${ }^{1,2} * \quad$ Pablo Figueroa ${ }^{1 \dagger} \quad$ Jean-Rémy Chardonnet ${ }^{2} \ddagger \quad$ Frédéric Merienne $^{2 \S}$ \\ José Tiberio Hernández ${ }^{1}$ ศ
}

${ }^{1}$ University of the Andes, Colombia $\quad{ }^{2}$ Institute Image, LE2I, Arts et Métiers, Univ. of Bourgogne-Franche-Comté, France

\begin{abstract}
This study presents a comparison of the influence of different VR environments in the task of selecting a preferred seat in an opera theater. We used gaze-based raycasting and headsets in a low-cost head-mounted display (HMD) (GearVR); and a virtual wand, head tracking, and headsets in a CAVE, two somewhat opposing technologies in the spectrum of current VR systems. Visual rendering and the selection technique depend on the capabilities of each environment, whereas the sound is approximated in both environments. Results show that subjects can select similar seats but their decisions differ between both environments. The results obtained can be useful in guiding the development of future VR applications.
\end{abstract}

Index Terms: I.3.7 [Computer Graphics]: Three-Dimensional Graphics and Realism-Virtual reality

\section{INTRODUCTION}

There are currently two main directions for VR development: PC-based and mobile-based. The capabilities of modern smartphones have motivated a new generation of enthusiastic VR developers. However, developing applications for this kind of platform is an important challenge. While newer devices are certainly providing more processing power than before, there are some limitations regarding the amount of detail that can be rendered. In contrast, PCbased applications can use the whole potential of graphics cards in desktops to render photo-realistic scenes for high-end technologies like Oculus Rift, HTC Vive and CAVE systems.

Mobile-based VR also has reduced natural interaction opportunities: currently tracking either the position of the head nor the users hands while maintaining at the same time the users motion freedom is not easy (without being tethered to a PC). Also, current mobile HMDs have a reduced field-of-view FOV (the amount of visual field visible at a given moment). On the other hand, highend displays like CAVE systems provide full body visualization, head tracking capabilities and a more natural FOV. However, some CAVE systems have a limited field-of-regard FOR (the amount of visual field visible physically rotating the head and body) compared with fully immersive HMDs (75\% for our four-sided CAVE).

The motivation of this study is getting insights concerning how much a VR experience could vary between these diametrically different technologies in terms of visual cues and interaction capabilities. Based on a 3D representation of a virtualized theater (Piccolo theater at Chalon-sur-Saone, France), we designed an application allowing users to select a seat based on their degree of visual and acoustic comfort. For the CAVE, we chose the magicwand metaphor [2] with raycasting as selection technique. It offers a more intuitive interaction by allowing subjects to use their own hand. For the GearVR system, we selected gaze-based raycasting as selection technique [7], a very popular method implemented for

\footnotetext{
*e-mail: jl.dorado59@uniandes.edu.co

†e-mail: pfiguero@uniandes.edu.co

‡e-mail:jean-remy.chardonnet@ensam.eu

$\S$ e-mail:frederic.merienne@ensam.eu

؟ e-mail:jhernand@uniandes.edu.co
}

this kind of devices. We designed the experiment so that subjects would be confronted mostly by the visual and interaction differences while the auditory variances were almost minimal. Figure 1 shows a comparison of rendering qualities of both environments.

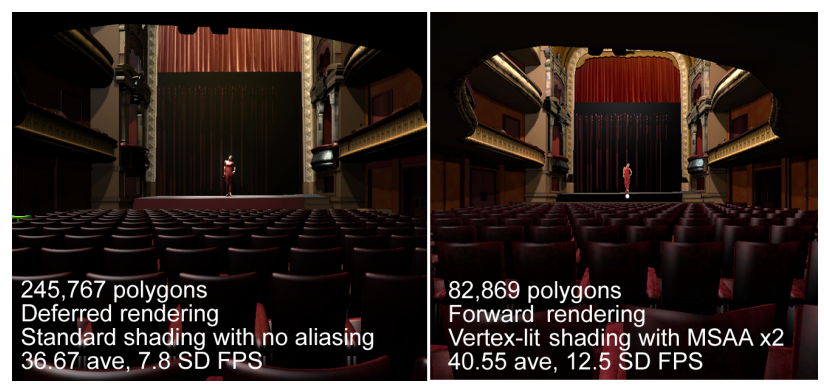

Figure 1: Final render quality. Left:CAVE, Right:GearVR.

\section{Related Work}

Virtual pointing or raycasting is one of the most widely implemented selection techniques in VR due to its good performance in comparison to other selection techniques [1]. We selected the most suitable raycasting technique for each environment according to the display capabilities. It has been shown that hand-based virtual pointing has some advantages for partially immersive projection displays when compared to full immersive displays [8]. Gazebased pointing seems to be more suitable for HMD systems [9]. We believe that both virtual pointing interactions will offer similar advantages for each display.

Regarding the differences between mobile-based HMD and CAVE systems, various reports show that the presence of head tracking and a greater FOV induce higher levels of presence [3]. Realism is an important condition for presence but recent evidence suggests that there is no direct correlation between realism and presence [6]. We believe that these visual cues will influence the seat preference making some seats more likely to be select. In a past experimental research about the importance of visual and auditory cues for an opera theater, auditory cues were more influential than visual cues in seat preference [5]. Based on the subjective assessments of this work, we performed a similar comparison but in this case considering the effect of VR displays.

\section{EXPERIMENT}

We focused our experimental design on evaluating the influence of the visual cues and interaction capabilities in seat selection. Both simulations were designed similarly so that the subjects have the same starting position and orientation. A static singer was located at the center of the stage with an opera song as audio source. In order to ensure that subjects perceived the same sound, subjects wear the same headphones. Also, the spatialization and reverberation were precalculated for both environments using Impulsonics API [4]. We performed a preliminary session for 2 minutes allow subjects to familiarize with the environment and the selection technique. Then, we asked them to find and select 5 seats consecutively based on a configuration defined a priori. We collected quantitative measures of their performance based on time and number of steps (moves).

After this task, we requested subjects to freely select different seats, but with the purpose of finding the seat offering the best quality in terms of visual and auditory appeal. Their selection was 
registered for comparative purposes. Finally, we used the subjective measures from [5] to evaluate their perceived quality. This assessment considers parameters such as perceived distance, brightness, apparent size, visual obstruction, visual comfort, sound clarity, loudness, envelopment and auditory comfort. This experiment was duplicated in two separated sessions for each VR display and we used a five-point Likert scale for all the measurements.

14 volunteers students (12 male, 2 female) participated in our experiment. All participants signed a consent reporting a normal vision condition and good health with no previous history of relevant diseases. We designed a within-subjects experiment where each subject tested both environments in separate sessions and with counterbalanced order among all users. Thus, subjects were randomly assigned to two groups according to their first assigned VR display. For each one, subjects must find the seats and select their favorite seat. For the first task, we provided two different sets of seats alternating their order between the groups to reduce possible biases. For the preference task, before the subjects tried to select their preferred seat in the second environment, we requested them to find their previously selected seat and decide whether it offered the same quality or they preferred to change. After each test, we took subjective measures of their perceived quality and realism.

\subsection{Hypothesis}

We believed that the differences in the visual cues would affect the seat preference judgment. In particular, subjects with a preference for seats located close to the stage will be influenced by the reduced visual quality in the GearVR. Also, we believed that subjects with a preference for the balcony will be affected by the lack of head position tracking or the reduced FOV. In this sense, we were interested in identifying which visual subjective measures are more influential in their decision according to the display capabilities.

We hypothesized that both techniques would have similar performance due their advantages for each display. Indeed the reduced FOV in the GearVR and the reduced FOR in the CAVE would affect the gaze-based and the magic-wand techniques respectively. Also, the lack of a wall behind in our CAVE would force subjects to select another seat and find a better view. In contrast, head position tracking offers advantages selecting occluded or distant seats.

\section{Results and Discussion}

As we expected, 10 of the 14 subjects changed their preference after the second session. Figure 2 shows the seat preference for each display technology and their corresponding selections performed between sessions. We noticed that the participants usually selected the same seats in both environments but their preference changed in the second session. The arrows indicate that most of the subjects did not perceive the same seat quality between environments. Contrary to our predictions, the visual quality was not influential and participants who preferred to be close to the stage in the CAVE did not tend to change their preference in the GearVR. In contrast, some subjects located in the balcony changed their preference to being closer to the stage with both display technologies. We believed that the causes are the lack of head position tracking combined with the reduction in FOV for CAVE-to-GearVR subjects. Overall the results did not show a clear tendency in seat preference.

A Wilcoxon Signed-Rank test show a significant difference in time ( $p=0.01)$ between the GearVR + gaze-based condition ( 23.31 ave, 12.42 SD) and the CAVE + magic-wand condition (33.65 ave, $19.33 \mathrm{SD}$ ). These results were caused mainly by issues with our CAVE implementation which causes that selecting a seat resulted more difficult. Interestingly, we did not find a significant difference in the number of steps, meaning that subjects learned quickly how to overcome the limited FOR in the CAVE. However, the selection technique performance could influence the preference judgment.

A paired sample t-test of the subjective measures identified 4 factors as relevant in the perceived seat quality: (1) realism, there is

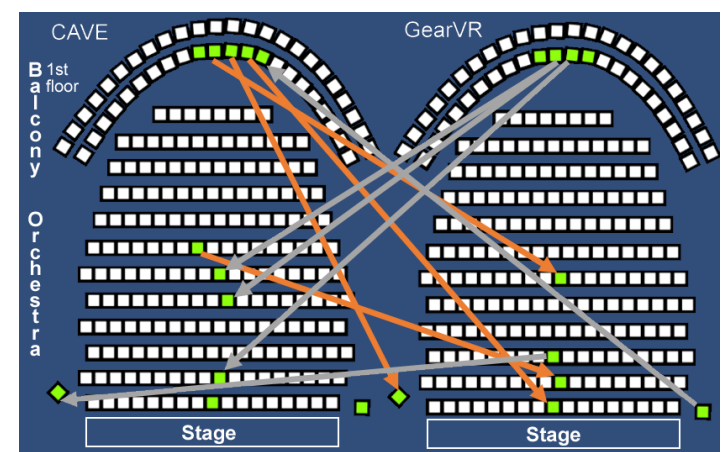

Figure 2: Seat preference and changes performed between sessions.

not significant differences in perceived realism between the lowquality GearVR version (3.92 ave, $1.14 \mathrm{SD}$ ) and the high-end CAVE version (4.21 ave, $1.48 \mathrm{SD}), t(13)=0.707, p=0.49$; (2) brightness, subjects perceived the stage in the CAVE as less bright (3.20 ave, $1.08 \mathrm{SD}$ ) than in the GearVR (3.86 ave, $0.63 \mathrm{SD}$ ), $t(13)=-2.092, p=0.055$; (3) apparent size, the FOV causes that subjects considered the stages dimension more comfortable in the CAVE (3.77 ave, 0.73 SD) than GearVR (3.25 ave, 0.75 SD) and (4) sound envelopment, subject perceived the sound slightly more realistic in the CAVE (3.33 ave, 0.81 SD) than in the GearVR (2.93 ave, $0.79 \mathrm{SD}), t(13)=-1.468, p=0.164$. These unexpected differences in auditory cues could be due an enhanced sense of presence.

The results seems indicating that the GearVR technological limitations do not affect substantially the subject's perceived seat quality but others associated display factors could affect their preference judgment. It would be ideal to control these factors generating similar conditions or imitating characteristics between environments with the purpose of analyzing the contribution of each factor in seat preference. Due the subjective nature of this research, we consider important to apply methods like pairwise comparison to reduce the subjectivity. Also, It would be interesting to compare the effects of using the same selection technique versus the most optimal selection technique and get more completed measures like accuracy and sense of presence. Finally, the next study will include a ground truth comparison with the real theater in terms of subject's expectations.

\section{REFERENCES}

[1] D. A. Bowman, D. B. Johnson, and L. F. Hodges. Testbed evaluation of virtual environment interaction techniques. Presence, 10(1):75-95, 2001.

[2] J. Ciger, M. Gutierrez, F. Vexo, and D. Thalmann. The magic wand. In Proceedings of the 19th spring conference on Computer graphics, pages 119-124. ACM, 2003.

[3] C. Hendrix and W. Barfield. Presence within virtual environments as a function of visual display parameters. Presence: Teleoperators \& Virtual Environments, 5(3):274-289, 1996.

[4] Impulsonics Phonon 3D. https://www.impulsonic.com, 2016.

[5] J. Y. Jeon, Y. H. Kim, D. Cabrera, and J. Bassett. The effect of visual and auditory cues on seat preference in an opera theater. The Journal of the Acoustical Society of America, 123(6):4272-4282, 2008.

[6] P. Khanna, I. Yu, J. Mortensen, and M. Slater. Presence in response to dynamic visual realism: a preliminary report of an experiment study. In Proceedings of the ACM symposium on Virtual reality software and technology, pages 364-367. ACM, 2006.

[7] T. Ohno and N. Mukawa. A free-head, simple calibration, gaze tracking system that enables gaze-based interaction. In Proceedings of the 2004 symposium on Eye tracking research \& applications, pages 115-122. ACM, 2004.

[8] A. Steed and C. Parker. Evaluating effectiveness of interaction techniques across immersive virtual environmental systems. Presence: Teleoperators and Virtual Environments, 14(5):511-527, 2005.

[9] V. Tanriverdi and R. J. Jacob. Interacting with eye movements in virtual environments. In Proceedings of the SIGCHI conference on Human Factors in Computing Systems, pages 265-272. ACM, 2000. 